\title{
Genome-scale cold stress response regulatory networks in ten Arabidopsis thaliana ecotypes
}

\author{
Pankaj Barah ${ }^{1}$, Naresh Doni Jayavelu², Simon Rasmussen ${ }^{3}$, Henrik Bjørn Nielsen ${ }^{3}$, John Mundy ${ }^{4}$ and Atle M Bones ${ }^{1 *}$
}

\begin{abstract}
Background: Low temperature leads to major crop losses every year. Although several studies have been conducted focusing on diversity of cold tolerance level in multiple phenotypically divergent Arabidopsis thaliana (A. thaliana) ecotypes, genome-scale molecular understanding is still lacking.
\end{abstract}

Results: In this study, we report genome-scale transcript response diversity of $10 \mathrm{~A}$. thaliana ecotypes originating from different geographical locations to non-freezing cold stress $\left(10^{\circ} \mathrm{C}\right)$. To analyze the transcriptional response diversity, we initially compared transcriptome changes in all 10 ecotypes using Arabidopsis NimbleGen ATH6 microarrays. In total 6061 transcripts were significantly cold regulated $(p<0.01)$ in 10 ecotypes, including 498 transcription factors and 315 transposable elements. The majority of the transcripts (75\%) showed ecotype specific expression pattern. By using sequence data available from Arabidopsis thaliana 1001 genome project, we further investigated sequence polymorphisms in the core cold stress regulon genes. Significant numbers of non-synonymous amino acid changes were observed in the coding region of the CBF regulon genes. Considering the limited knowledge about regulatory interactions between transcription factors and their target genes in the model plant $A$. thaliana, we have adopted a powerful systems genetics approach- Network Component Analysis (NCA) to construct an in-silico transcriptional regulatory network model during response to cold stress. The resulting regulatory network contained 1,275 nodes and 7,720 connections, with 178 transcription factors and 1,331 target genes.

Conclusions: A. thaliana ecotypes exhibit considerable variation in transcriptome level responses to non-freezing cold stress treatment. Ecotype specific transcripts and related gene ontology (GO) categories were identified to delineate natural variation of cold stress regulated differential gene expression in the model plant $A$. thaliana. The predicted regulatory network model was able to identify new ecotype specific transcription factors and their regulatory interactions, which might be crucial for their local geographic adaptation to cold temperature. Additionally, since the approach presented here is general, it could be adapted to study networks regulating biological process in any biological systems.

Keywords: Arabidopsis thaliana, Ecotypes, Cold stress, Natural variation, Adaptation, Gene expression, Regulatory networks, Arabidopsis thaliana 1001 genome, Systems biology, Network component analysis

\section{Background}

Being sessile organisms, plants have evolved strategies to survive in unfavourable environmental conditions. Intraspecific variation in response to environmental stresses is clearly visible among plant species [1-4]. Understanding the molecular basis of such local adaption to complex environmental conditions has proven to be very useful in

\footnotetext{
* Correspondence: atle.bones@bio.ntnu.no

${ }^{1}$ Department of Biology, Norwegian University of Science and Technology, Trondheim N-7491, Norway

Full list of author information is available at the end of the article
}

selecting better traits or target genes for modern plant sciences [5]. Cold stress is a naturally occurring hazard to world crop production. Cold stress contributes to poor germination, stunted seedlings, chlorosis, reduced leaf expansion and wilting, and may also lead to death of tissue (necrosis) [6]. Exposure to cold stress also slows down the reproductive development of plants. Plants perceive cold by the receptor at the cell membrane and a signal is initiated to activate the cold-responsive genes and transcription factors for mediating stress tolerance $[7,8]$. The CBF cold response pathway has a major role in cold response, 
tolerance and acclimation; however, considerable differences in the sets of cold regulated genes were observed [9]. $C B F$ genes are induced after just few minutes of cold exposure. They encode a small family of transcription factors known as CBF1, CBF2, and CBF3 (also known as DREB1B, DREB1C and DREB1A). Cold induction of CBF genes regulates a set of about 100 downstream genes. Among them, the immediate target genes of CBF1-3 include CRT (C-repeat)/DRE (dehydration responsive element) elements in their promoter regions. CBF1-3 proteins bind to this DNA regulatory sequence. The dehydrationresponsive element (DRE) is also known as low temperature response element (LTRE), which contributes to cold responsiveness [10]. Interestingly, induction of the CBF regulon enhances both cold and drought tolerance [11]. Earlier transcriptome profiling studies have shown that multiple regulatory pathways are activated in A. thaliana during cold exposure in addition to the $\mathrm{CBF}$ cold-response pathway [12].

Natural variation for cold response and tolerance is an important element of adaptation and geographic distribution of plant species. There is clear association between plasticity of gene expression and adaptability of an organism [13]. There have been several studies focusing on diversity of cold tolerance level in multiple phenotypically divergent $A$. thaliana ecotypes [14-16]. McKhann et al. reported that CBF and COR (Cold Regulated) genes respond differently to cold stress in eight accessions, though they could not find clear correlation between gene expression, sequence polymorphism and cold tolerance [17]. However, the molecular basis of the natural variation during cold stress response in plants at genome scale is not fully understood yet.

Transcriptional profiling has become a major tool to identify genes exhibiting transcriptional regulation in plants as an effect of changing environmental conditions taking Arabidopsis as a model system [18]. Variation in experimental conditions and protocols makes it difficult to extract and compare information from data sets produced by individual laboratories [19]. To overcome such problems, we subjected 10 ecotypes of $A$. thaliana to 5 individual stress treatments and 6 combinations of these stress treatments under the same experimental set up and profiling protocols [20]. We have considered all the cold experiments conducted on 10 ecotypes from this already published dataset (GEO accession GSE41935), to explore genome-scale transcriptomic response signatures of $A$. thaliana during cold stress treatment. By utilising data available from recently published $A$. thaliana 1001 genome project, we further analysed sequence polymorphisms in the CBF regulon genes [21].

It is likely that differential expressions or variation in mRNA stability caused by coding sequence polymorphisms significantly contribute to natural variation in
A. thaliana [22]. Information about differentially regulated genes during different stress conditions is often available as an outcome of microarray experiments. However, in many cases, little is known about the regulation and interaction of these genes [23]. Being highly dynamic in nature, any biological system continuously changes responding to environmental and genetic perturbations. Differential dynamic network mapping of facilitates the exploration of previously unknown interactions [24]. While the A. thaliana genome has 1922 TFs [25], experimentally confirmed regulatory relations are available for less than 100 TFs only (as per information extracted from the AGRIS database, version updated on September 10th, 2012) [26]. Tirosh et al. explained how regulatory relationships can also be deduced from patterns of evolutionary divergence in molecular properties such as gene expression [27]. To compensate for the lack of information on transcription factor activity at the genome scale, several computational algorithms have been developed to identify regulatory modules and their condition-specific regulators from gene expression data [28-30]. Network Component Analysis (NCA) is such an approach, which has been successfully implemented in several species including $A$. thaliana, to determine both activities and regulatory influences for a set of transcription factors on target genes in various perspectives [31-33]. By taking the advantage of the NCA method, we predict ecotype specific regulatory relationships, which provide new information towards understanding the natural variation in cold response pattern among different ecotypes of the model plant A. thaliana.

\section{Methods}

\section{Microarray data}

We have considered all the cold stress microarray experiments conducted on 10 ecotypes during the ERA-PG Multi-stress project [20], to explore genome-scale transcriptomic response signatures of $A$. thaliana during cold stress treatment (microarray data available at GEO with the accession GSE41935). All the experiments of ERA-PG Multistress project were set up in environmentally controlled rooms at the plant growth facilities at RISØ DTU National Laboratory for Sustainable Energy (Roskilde, Denmark). A pilot study using wild type plants $\mathrm{Col}$ and Ler was set up to find the appropriate conditions at sublethal doses [20]. These initial observations indicated that an optimal time before the onset of a phenotypic response (e.g.: wilting, dehydration) while avoiding tissue damage was 3 hours. Ten $A$. thaliana wild ecotypes (Table 1 ) were grown in soil under long day photoperiod and $24^{\circ} \mathrm{C}$ in a greenhouse setting for one generation to amplify homogeneous seed for all different genotypes. The obtained seeds were sown into trays and grown in a Conviron growth chamber (Winnipeg, Manitoba, Canada) under a $12 \mathrm{hr} /$ 
Table 1 Summary of the ecotypes and their gene expression pattern during cold treatment

\begin{tabular}{|c|c|c|c|c|c|c|c|c|}
\hline \multirow[b]{2}{*}{$\begin{array}{l}\text { Ecotype } \\
\text { name }\end{array}$} & \multicolumn{2}{|l|}{ Ecotypes } & \multicolumn{6}{|c|}{ Differentially regulated transcripts } \\
\hline & ${ }^{*}$ Geographic origin & $\begin{array}{l}\text { Latitude } \\
\text { ('North) }\end{array}$ & Total & $\begin{array}{l}\text { Total } \\
\text { up }\end{array}$ & $\begin{array}{l}\text { Total } \\
\text { down }\end{array}$ & $\begin{array}{l}\text { Unique } \\
\text { total }\end{array}$ & $\begin{array}{l}\text { Unique } \\
\text { up }\end{array}$ & $\begin{array}{l}\text { Unique } \\
\text { down }\end{array}$ \\
\hline Cvi & Cape Verdia Islands & 16 & 2004 & 603 & 1401 & 1230 & 276 & 954 \\
\hline Kas-1 & Kashmir, India & 34 & 1097 & 487 & 610 & 442 & 153 & 289 \\
\hline Куо-2 & $\begin{array}{l}\text { Kyoto city, western part of Hoshu Island, } \\
\text { Japan }\end{array}$ & 35.5 & 877 & 458 & 419 & 305 & 104 & 201 \\
\hline Sha & Shakdara, Tadjikistan & 39 & 620 & 215 & 405 & 268 & 70 & 198 \\
\hline Col-0 & Columbia, United States & 38.5 & 185 & 120 & 65 & 89 & 48 & 41 \\
\hline Kond & Kondara, Tadjikistan & 38.8 & 814 & 428 & 386 & 384 & 167 & 217 \\
\hline $\mathrm{C} 24$ & Coimbra, Portugal & 40 & 1427 & 931 & 496 & 758 & 460 & 208 \\
\hline Ler & Landsberg, Poland & 48 & 619 & 195 & 424 & 348 & 91 & 257 \\
\hline$A n-1$ & Antwerpern, Belgium & 51.5 & 632 & 308 & 324 & 188 & 69 & 119 \\
\hline Eri & Erigsboda, Sweden & 56 & 967 & 804 & 163 & 541 & 427 & 114 \\
\hline
\end{tabular}

*Geographic origins of the ecotypes were collected from the donor, Arabidopsis 1001 Genome project database [75] and Swindell et al. [13].

This table represents geographic distribution of the $10 \mathrm{~A}$. thaliana ecotypes and number of cold regulated genes in each of the ecotypes ( $\mathrm{p} \leq 0.01$ ). Up and down regulation was calculated based on fold change ratios compared to respective untreated controls in individual ecotypes. Ecotype Col-0, which is a cold tolerant ecotype have very less number of cold regulated transcripts compare to others. On the other hand Cvi, the southernmost accession from a warm climate, exhibits more number of cold responsive transcripts. (Unique $=$ Unique to the respective ecotype).

$12 \mathrm{hr}$ photoperiod, $24^{\circ} \mathrm{C}$ and standard $A$. thaliana growth conditions. Three week-old plants were then placed for three hours into the environmentally controlled growth rooms that were preset to cold stress conditions $\left(10^{\circ} \mathrm{C}\right)$. Triplicated (biological) trays with the wild type controls were subject to the cold treatment. After the stress treatments, leaves tissue were collected and promptly frozen in liquid nitrogen for subsequent microarray experiments.

\section{Statistical analysis of the data}

Resulting data from the microarray experiments was pre-processed using the RMA [34] implementation in the oligo package [35] in $\mathrm{R}$ programming platform [36]. Gene annotation was acquired from TAIR10 [37] using biomaRt data mining tool [38]. Differentially expressed genes between control and treated plants were identified using t-test $(\mathrm{p}<0.01)$. Genotype specific responses to stress were identified by the interaction effect from a two-way ANOVA $[39,40]$ of the genotype and treatment effect $(p<0.01)$. The union of stress responsive genes was further used for network-based analysis. Heat maps were plotted using TM4 microarray software suite [41].

\section{Gene set enrichment analysis (GSEA)}

The Biological Networks Gene Ontology (BiNGO) tool [42], an open-source Java tool was used to determine which Gene Ontology (GO) terms [43] that were significantly overrepresented in our differentially regulated gene lists ( $\mathrm{p}$-values were Bonferroni corrected).

\section{Sequence analysis}

Sequences for CBFs, and COR genes were downloaded from A. thaliana 1001 Genome project (http://signal. salk.edu/). Initially sequences from all available ecotypes in the 1001 genome database (706) were downloaded, but incomplete sequences were discarded before the analysis. Apart from the coding regions, we have considered 1,000 bp upstream sequences for alignment. All positions containing gaps and missing data were eliminated. Multiple sequence analysis performed using Clustal w [44]. Tajima's D [45] statistical test to identify sequences which do not fit the neutral theory model at equilibrium between mutation and genetic drift were performed using MEGA5 suit [46].

Network component analysis and network reconstruction Network component analysis is a computational method for reconstructing the hidden regulatory signals (TFAs) from gene expression data with known connectivity information in terms of matrix decomposition [31,47]. NCA model assumes the log-linear relationship between target genes expression profiles and TFAs:

$$
\frac{E_{i}(t)}{E_{i}(0)}=\Pi_{j=1}^{L}\left(\frac{T F A_{j}(t)}{T F A_{j}(0)}\right)^{C S_{i j}}
$$

Where $E_{i}(t)$ and $E_{\mathrm{i}}(0)$ are the expression values of gene $\mathrm{i}$ at different measurement conditions and reference point 0 , and similarly $T F A_{j}(\mathrm{t})$ and $T F A_{j}(0)$ are the activities of $T F_{j}$, and $C S_{i j}$ represents the control strength of TF $j$ on gene $i$. Taking logarithms, the equation (1) becomes:

$$
\log [E r]=[C S] \log [T F A r]
$$

Where the matrix $E r$ represents the expression values of genes at different measurement conditions, matrix $C S$ is the control strength of each TF on each TG and TFAr 
represents the TFAs of all the TFs. The dimensions of $[E r]$ is $\mathrm{N} \times \mathrm{M}(\mathrm{N}$ is the number of genes and $\mathrm{M}$ is the number of measurement conditions), $[C S]$ is $\mathrm{N} \times \mathrm{L}$ ( $\mathrm{L}$ is the number of TFs), and for [TFAr] is $\mathrm{L} \times \mathrm{M}$. We can further simplify the above equation (2) as:

$$
[E]=[C][T]
$$

Here expression matrix $[\mathrm{E}]$ corresponds to $[\mathrm{Er}]$ in equation (2), connectivity strength matrix $[\mathrm{C}]$ is equivalent to $[\mathrm{CS}]$ and transcription factor activity matrix $[\mathrm{T}]$ corresponds to $\log [\mathrm{TFAr}]$ in equation (2). Based on above formulation, the decomposition of $[\mathrm{E}]$ into $[\mathrm{C}]$ and $[\mathrm{T}]$ can be achieved by minimizing the following objective function:

$$
\min \|([E]-[C][T])\|
$$

$$
\text { s.t. } C \in Z 0
$$

Here Z0 is the initial connectivity pattern. The estimation of $[\mathrm{C}]$ and $[\mathrm{T}]$ is performed by using a two-step leastsquares algorithm and normalized through a nonsingular matrix $[\mathrm{S}]$ according to,

$$
[E]=[C][T]=[C][S]\left[S^{-1}\right][T]
$$

In order to guarantee uniqueness of the solution for the equation (4) up to a scaling factor, there are certain criteria to be satisfied termed as NCA criteria: (a) The connectivity matrix $[\mathrm{C}]$ must have full-column rank. (b) When a node in the regulatory layer is removed along with all of the output nodes connected to it, the resulting network must be characterized by a connectivity matrix that still has full-column rank. (c) T matrix must have full row rank.

The algorithm for NCA analysis is implemented in MATLAB by Liao and his colleagues and it is available online for download (http://www.seas.ucla.edu/ liaoj/ downloads.html). With NCA as reconstruction method, we predicted significant TFs and connectivity strength on target genes and TFAs of TFs.

\section{Results and discussion}

\section{Different transcriptome signatures of 10 Arabidopsis}

\section{ecotypes responding to cold stress}

To cover a wide array of phenotypic variation, 10 natural accessions of $A$. thaliana representing habitats from $16^{\circ}$ to $56.5^{\circ}$ northern latitude were selected during the ERA-PG Multi-stress project. These accessions or ecotypes were- Cvi (Cape Verde Islands), Kas-1 (Kashmir, India), Kyo-2 (Kyoto, Japan), Sha (Shakdara, Tadjikistan), Col-0 (Columbia, USA), Kond (Kondara, Tadjikistan), C24 (Coimbra, Portugal), Ler (Landsberg, Poland), An-1 (Antwerpe, Belgium), Eri (Erigsboda, Sweden) (details in Table 1). We have chosen cut-off $\mathrm{p} \leq 0.01$ to define a gene as differentially regulated. Using the results from these ten ecotypes, we were able to examine the transcriptional differences that occurred during early hours of cold treatment. The results (Table 1) indicated that A. thaliana ecotypes have visibly different transcriptome signatures in response to cold stress. Variable numbers of transcripts were up or down regulated by cold stress. Considering the two extreme ecotypes, Col-0 being known as cold tolerant ecotype had significantly less number of differentially regulated transcripts, while Cvi being the southernmost ecotype (among the 10 used in our experiments) had the highest number of differentially regulated transcripts. Ecotype Cvi (Cape Verde Islands) was associated with a climate temperature comparatively higher than that of the ecotype Col-0, and fact was well reflected in their transcriptional responses to cold treatment. Similar results were also reported by earlier [13].

A unified list of 6061 cold regulated transcripts $(\mathrm{p}<$ 0.01 ) was generated from all the 10 ecotypes (Additional file 1). The total number of differentially regulated TFs in this list was 498. Interestingly, 4553 (75\%) transcripts were differentially regulated only in one of the ten ecotypes. The significant list of differentially regulated transcripts includes most of the known cold regulated genes. Figure 1 displays fold change values (treatment vs. control) calculated from normalized expression index for top 1000 significant genes from all the 10 ecotypes. Global observation of the heat map indicates differentially regulated transcriptome signatures in response to nonfreezing cold treatment in ten different $A$. thaliana ecotypes. Hierarchical clustering $(\mathrm{HCL})$ was performed with Pearson correlation using average linkage method and 10,000 bootstrapping for the top 1000 cold regulated transcripts based on fold change ratios with respects to their respective controls. Ecotype Col-0 is distinctly separated out from others. Southern ecotypes Cvi, Sha and Kyo2 were grouped closely. Zhen et al. [16] has reported earlier a positive correlation between freezing tolerance and latitude of origin, based on physiological data collected from $71 \mathrm{~A}$. thaliana ecotypes. Hannah et al. [48] used 9 accessions of $A$. thaliana to show that cold tolerance of natural accessions correlates with habitat winter temperatures. Clustering of the gene expression pattern in response to non-freezing cold stress exposure in ten ecotypes during our analysis doesn't reflect a clear latitudinal trend.

\section{Ecotype specific cold regulated transcript lists contain many transcription factors (TFs) and transposable elements (TEs)}

In contrast to the relatively small number of transcripts with altered expression shared by all the ten ecotypes, majority of the transcripts (75\%) showed ecotype specific expression pattern (Additional file 2). Each of the ecotypes had unique sets of differentially regulated transcripts in 


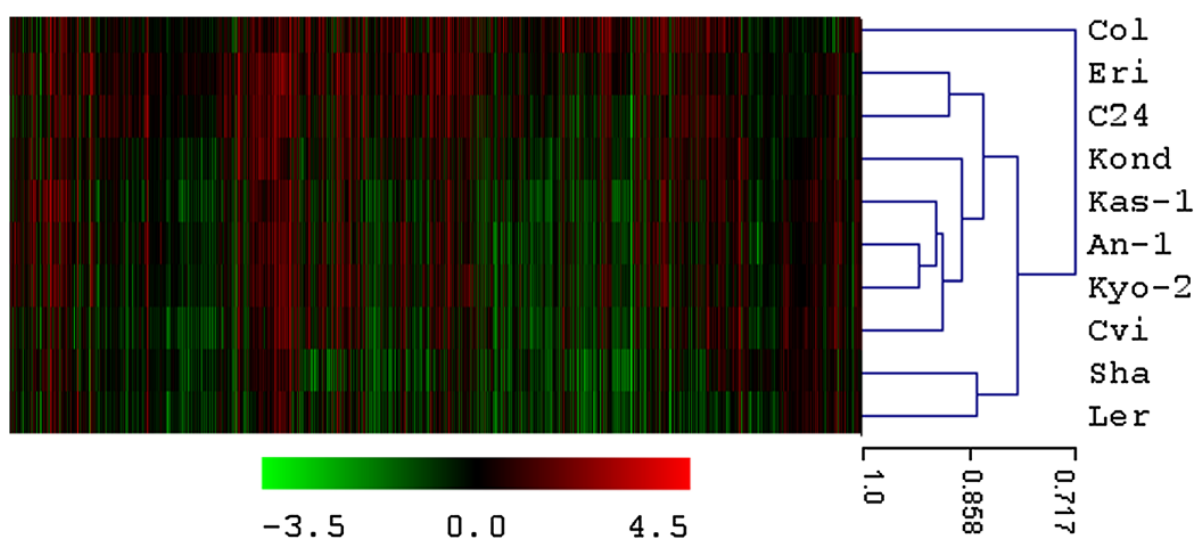

Figure 1 Heat map visualization of the cold transcriptome of the ten ecotypes. The heat map visualizes hierarchical clustering (with Pearson's correlation coefficient using average linkage method) of top 1000 cold regulated transcripts based on gene expression fold change ratios compared to their respective controls from 10 different ecotypes. Genes are shown as columns and ecotypes are shown as rows. As a global observation, this heat map indicates differential regulation signatures in response to non-freezing cold treatment in different A. thaliana ecotypes. Cold tolerant ecotype Col-0 ecotype is distinctly separated out from others.

response to cold stress. From the list of differentially regulated transcripts, it was found that 498 encoded for Arabidopsis TFs and 320 TFs ( 64\% of all the differentially regulated TFs) were differentially regulated in single ecotypes. The ecotype specific differentially cold regulated TFs are listed in Table 2. The list of differentially regulated transcripts includes many well-known cold regulators like $C B F \mathrm{~s}$, DREB1A, DREB1B, DREB2B, RAV1, ERF2, and ERF5. We have surveyed existing available transcription factor - target gene (TF-TG) regulatory interactions available in public databases and literature. There were 59 TFs reported as associated to cold responses in GO (Gene Ontology) database and TAIR (The Arabidopsis Information Resources). Unfortunately, none of them were included in the AtRegNet server which contained experimentally validated regulatory interactions for only 69 out of nearly 1922 known Arabidopsis TFs.

Nimblgen12-plex Arabidopsis microarray chip included 3822 transposable element (TE) probes. We have observed 315 TEs ( 10\% of the total TE probes printed on the chip) in the ecotype specific differentially regulated transcript list. The distribution of the differentially regulated TEs in ten ecotypes were as follows - Col-0 (21), Ler (81), Cvi (71), Eri (31), Kas2 (16), Kond (39), Kyo2 (23), C24 (15), Sha (22) and An1 (8). Somatic events, in particular, the activity of transposable elements (TEs) do play an important role in plant genome evolution [49]. Lee et al. [50] reported that coldregulated gene expression was not only controlled transcriptionally, but might also be regulated at the posttranscriptional and chromatin level [50]. A change in the epigenetic state of TEs by cold stress might contribute to regulatory activities for adjacent genes [51]. Recently Wang et al. has demonstrated that both TE sequence polymorphisms and presence of linked TEs are positively correlated with intraspecific variation in gene expression [52]. Some of the differentially regulated TEs in our cold experiments might potentially be interesting targets to explore diversity of cold stress responses among different $A$. thaliana ecotypes. Further targeted experiments in this direction can explore the molecular level details of any potential role of these TEs on genomic adaptation of the ecotypes to their local environment.

\section{Gene set enrichment analysis indicates activation of common and unique processes in different ecotypes}

To investigate functionally relevant changes, gene ontology based overrepresentation analysis was performed using BinGO software considering the up-regulated gene lists from each of the ten ecotypes (Additional file 3). From this analysis we have created a GO attribute table by uniting all the statistically significant overrepresented GO categories from each of the ten ecotypes (Additional file 4 ). Genes showing significant variation in mRNA expression level in $A$. thaliana during different stress conditions mainly belong to categories like signal transduction, transcription and stress response [53]. This reflects the potential variations in the regulatory mechanisms of these genes among different ecotypes. Apart from common cold stress responsive categories like- response to cold stress, response to low temperature, cold acclimation etc., we observed few other biological processes to be differentially up-regulated in various ecotypes (Table 3 ). Some of the interesting and top GO categories were as follows.

\section{Cold response is coupled with light stimulus}

Along with the general cold response pathways or processes, there were several overrepresented categories related to 'response to light'. Few genes in these categories were as follows- At1g29920 (LHCB1), At5g24470 (PRR5), 
Table 2 Cold regulated transcription factors

\begin{tabular}{ll}
\hline Ecotype & Unique TFs (Up regulated) \\
\hline Cvi & ANAC014, ANAC042, ANAC058, AtHB24, AtHB32, AtMYB103, \\
& HEC1, HSFB2A, LBD27, LBD35, LD, PIL6, SRL2, At3g20880, At \\
& At1g09060, At3g11450, At5g45270 \\
& \\
& \\
Kas.1 & AtGRF3, BPC6, HAt3, HSFA8, IAA29, SSL2, SWN, WRKY3, WRKY \\
& WRKY66, At3g45260, At1g67310, At2g45460 \\
Kyo.2 & AtIDD2, CAL1, HAt14, LCL1, PHE2, RAP2.9, SNZ, SPL3, TOC1, \\
& At4g18870, At5g51790 \\
Col-0 & At3G50750, At2G27630, At5G22990, At1G48195, At4G37850
\end{tabular}

Col-0 At3G50750, At2G27630, At5G22990, At1G48195, At4G37850
Unique TFs (Down regulated)

AIL6, ANAC041, ANAC074, ANAC103, ARR2, ASML2, AtHB23,

AtMYB11, AtMYB17, AtMYB86, AtNAC3, AtY13, bt5, EMB3008, ETC1, GNC, HAt22, HDG12, IAA18, LBD23, MAF4, MYB113, MYB3, MYB33, MYB92, MYC6.2, NTL9, PAN, PCF1, PUX2, SDG40, SGR1, SPL5, SUVR4, tcp17, TCP3, TGA6, VND1, WOX13, WRKY50, At1g16640, At3g06160, At4g34400, At4g00940, At5g49300, At3g57480, At5g10970, At2g05160, At5g40880, At3g16940, t5g38140, At2g20110, At1g07520, At1g63100, At1g44810, At4g00232, At4g26170, At1g09710, At1g33420, At2g01810, At3g53370, At5g51910, At1g76870, At1g26260, At1g62975, At4g00870, At4g14410, At4g29930, At5g46830, At5g65320

AL1, BT4, DUO1, GBF6, HSFB1, HSFB4, MNP, TED5, TIFY3B, U2AF35B, ZBF1, ZFN3, ZFP4, At5G52020, At5G06770, At5G41920, At4G22140, At5G50670, At1G03040, At3G23690

HDT3, PMG1, TRFL6, UNE12, WER1, At5g61190, At4g23800, At2g45800, At1g69170, At1g68920, At2g46510

ACD6, ACS3, CYP71A28, CYP81K1, MEA, MLP28, MYB24, PSRP5, RCK, STR16, XIJ, At3g21570, At5g33260, At2g21930, At5g26930, At5g15620, At3g18840, At1g31370, At2g01031, At2g09850, At2g24930, At1g79120, At4g09070, At3g56600, At5g39150, At2g35300, At1g23570, At5g02140, At1g23060, At3g14750, At1g27300, At3g16840, At3g03920, At2g07671, At1g53060, At5g66230, At5g58570, At5g26690, At1g27330, At1g18720, At5g18850

ADOF2, ANAC097, AtHB27, BME3-ZF, bZIP61, GIF2, HAP3A, HAP5B, ING1, RAP2.11, TGA1, At3g51080, At2g40970, At5g47390

AGL24, AGL43, CRF1, ETT, IAA7, LBD38, PHB-1D, PTAC1, SRS8, ZFHD2, At5g02460, At5g41030

ANAC065, AtHB16, AtMYB63, BPC5,HSFB2B, LBD22, RGA2, SCL3, ZFHD3, At1g49475, At1g68520, At4g24060, At3g24050, At4g14540, At2g44730, At2g21235 RVE2, SPL1R2, SSRP1,SUVH3, WRKY26, WRKY33, WRKY40, WRKY55, At5g51190, At2g17410, At4g17570, At1g26610, At4g15420, At5g26610, At5g12440, At3g52250, At5g06110, At1g20640, At1g64530, At2g18090, At2g37520, At1g01260, At5g57150

Ler ddf2, HSFC1

An.1 AGL56, RD26, SOC1, SUVH1, tify5a, At1g79700, At4g15250, At2g42150,

Eri ALC, ANAC011, ANAC019, ANAC044, ANAC046, AtAF2, AtNAC3, AZF2, BPC4, COL9, DAG2, ERF5, ERF8, IAA17, IAA5, MYB59, MYBR1, RAP2.10, SHN3, SHY1, TIFY10B, WRKY22, WRKY27, WRKY28, ZFHD4, At4g01580, At2g40340, At2g40350, At4g32800, At2g45050, At3g49930, At3g60580, At3g08505, At3g14020, At1g25550, At3g12730, At5g01200, At5g05790, At3g21210, At3g53680, At2g18850, At3g21330, At3g23210, At1g19490

List of differentially cold regulated (up and down) TFs, unique for each of the 10 A. thaliana ecotypes (significance threshold $p \leq 0.01$ ).

At4g08920 (OOP2), At1g02340 (RSF1), At1g06040 (STO), At3g27690 (LHCB2.4), At3g54720 (PT), At2g42540 (COR15A), At2g26990 (FUS12), At5g24120 (SIGE), At2g 46970 (PIL1), At4g18130 (PHYE), At5g67030 (ZEP), At5g 45340 (CYP707A3), At1g02400 (GA2OX6), At2g46790 (TL1), At3g28860 (PGP19) At2g46340 (SPA1), At4g19230 (CYP707A1), At2g18790 (PHYB). Light and cold signals is known to integrate and cross talk for cold tolerance, via a CBF and ABA-independent pathway [54]. Franklin et al. [55] investigated the modulation of low R/FR signalling by ambient temperature and results showed that a low red to far-red ratio (R/FR) light signal increases $\mathrm{CBF}$ gene expression in A. thaliana in a manner dependent on the circadian clock. Red or far-red light signalling pathway is one of the significantly up-regulated GO categories in some of the ecotype [55]. Such signals stimulate expression of $C B F$ genes through ambient temperaturedependent coupling of $\mathrm{CBF}$ transcription factors to downstream COLD REGULATED (COR) genes.

\section{Chlorophyll biosynthetic process}

Another overrepresented GO category was chlorophyll biosynthetic process which included several genes like At1g78600 (STH3), At5g54190 (PORA), At3g59400 (GUN4), 
Table 3 GO terms attribute matrix from the significantly regulated gene-list for each ecotype, generated using BiNGO software

\begin{tabular}{|c|c|c|c|c|c|c|c|c|c|c|}
\hline GO-terms & An.1 & Col -0 & Cvi & Eri & Kas.1 & Kond & Куо. 2 & Ler & $\mathrm{C} 24$ & Sha \\
\hline Response to abiotic stimulus & $\checkmark$ & $\checkmark$ & $\checkmark$ & $\checkmark$ & $\checkmark$ & $\checkmark$ & $\checkmark$ & $\checkmark$ & $\checkmark$ & $\checkmark$ \\
\hline Response to chemical stimulus & $\checkmark$ & $\checkmark$ & $\checkmark$ & $\checkmark$ & $\checkmark$ & $\checkmark$ & $\checkmark$ & $\checkmark$ & $\checkmark$ & $\checkmark$ \\
\hline Response to cold & $\checkmark$ & $\checkmark$ & $\checkmark$ & $\checkmark$ & $\checkmark$ & $\checkmark$ & $\checkmark$ & $\checkmark$ & $\checkmark$ & $\checkmark$ \\
\hline Response to organic substance & $\checkmark$ & $\checkmark$ & $\checkmark$ & $\checkmark$ & $\checkmark$ & $\checkmark$ & $\checkmark$ & $\checkmark$ & $\checkmark$ & $\checkmark$ \\
\hline Response to endogenous stimulus & $\checkmark$ & $\checkmark$ & $\checkmark$ & $\checkmark$ & $\checkmark$ & $\checkmark$ & $\checkmark$ & $\checkmark$ & $\checkmark$ & $\checkmark$ \\
\hline Response to hormone stimulus & $\checkmark$ & $\checkmark$ & $\checkmark$ & $\checkmark$ & $\checkmark$ & . & $\checkmark$ & $\checkmark$ & $\checkmark$ & $\checkmark$ \\
\hline Circadian rhythm & $\checkmark$ & $\checkmark$ & $\checkmark$ & $\checkmark$ & $\checkmark$ & . & $\checkmark$ & $\checkmark$ & $\checkmark$ & $\checkmark$ \\
\hline Response to light stimulus & $\checkmark$ & . & $\checkmark$ & $\checkmark$ & $\checkmark$ & & $\checkmark$ & $\checkmark$ & $\checkmark$ & $\checkmark$ \\
\hline Response to water & $\checkmark$ & $\checkmark$ & . & $\checkmark$ & $\checkmark$ & . & $\checkmark$ & $\checkmark$ & $\checkmark$ & $\checkmark$ \\
\hline Response to jasmonic acid stimulus & $\checkmark$ & & $\checkmark$ & $\checkmark$ & . & $\checkmark$ & $\checkmark$ & $\checkmark$ & $\checkmark$ & $\checkmark$ \\
\hline Response to water deprivation & $\checkmark$ & $\checkmark$ & . & $\checkmark$ & $\checkmark$ & & $\checkmark$ & $\checkmark$ & $\checkmark$ & $\checkmark$ \\
\hline Response to red or far red light & $\checkmark$ & & $\checkmark$ & $\checkmark$ & $\checkmark$ & . & $\checkmark$ & $\checkmark$ & $\checkmark$ & $\checkmark$ \\
\hline Cold acclimation & $\checkmark$ & & . & $\checkmark$ & $\checkmark$ & $\checkmark$ & $\checkmark$ & $\checkmark$ & $\checkmark$ & $\checkmark$ \\
\hline Rresponse to other organism & $\checkmark$ & . & $\checkmark$ & . & $\checkmark$ & $\checkmark$ & $\checkmark$ & $\checkmark$ & $\checkmark$ & $\checkmark$ \\
\hline Response to blue light & $\checkmark$ & . & $\checkmark$ & $\checkmark$ & $\checkmark$ & . & $\checkmark$ & $\checkmark$ & $\checkmark$ & $\checkmark$ \\
\hline Response to abscisic acid stimulus & $\checkmark$ & . & $\checkmark$ & & $\checkmark$ & $\checkmark$ & $\checkmark$ & . & $\checkmark$ & $\checkmark$ \\
\hline Response to far red light & $\checkmark$ & . & $\checkmark$ & $\checkmark$ & $\checkmark$ & . & $\checkmark$ & $\checkmark$ & $\checkmark$ & . \\
\hline Multi-organism process & $\checkmark$ & . & $\checkmark$ & & $\checkmark$ & $\checkmark$ & $\checkmark$ & . & $\checkmark$ & $\checkmark$ \\
\hline Response to red light & $\checkmark$ & . & $\checkmark$ & $\checkmark$ & $\checkmark$ & . & $\checkmark$ & $\checkmark$ & $\checkmark$ & . \\
\hline Response to fungus & $\checkmark$ & . & $\checkmark$ & & $\checkmark$ & . & $\checkmark$ & $\checkmark$ & $\checkmark$ & $\checkmark$ \\
\hline Response to carbohydrate stimulus & $\checkmark$ & . & . & $\checkmark$ & $\checkmark$ & $\checkmark$ & $\checkmark$ & & $\checkmark$ & $\checkmark$ \\
\hline Regulation of transcription & $\checkmark$ & & & $\checkmark$ & $\checkmark$ & . & $\checkmark$ & . & $\checkmark$ & $\checkmark$ \\
\hline Regulation of macromolecule biosynthetic process & $\checkmark$ & . & . & $\checkmark$ & $\checkmark$ & . & $\checkmark$ & & $\checkmark$ & $\checkmark$ \\
\hline Regulation of nucleobase, acid metabolic process & $\checkmark$ & . & . & $\checkmark$ & $\checkmark$ & . & $\checkmark$ & & $\checkmark$ & $\checkmark$ \\
\hline Pigment biosynthetic process & $\checkmark$ & . & $\checkmark$ & . & . & $\checkmark$ & $\checkmark$ & $\checkmark$ & $\checkmark$ & 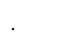 \\
\hline Regulation of biosynthetic process & $\checkmark$ & . & . & $\checkmark$ & $\checkmark$ & . & $\checkmark$ & & $\checkmark$ & $\checkmark$ \\
\hline Regulation of nitrogen compound metabolic process & $\checkmark$ & . & . & $\checkmark$ & $\checkmark$ & & $\checkmark$ & & $\checkmark$ & $\checkmark$ \\
\hline Regulation of gene expression & $\checkmark$ & . & & $\checkmark$ & $\checkmark$ & . & $\checkmark$ & . & $\checkmark$ & $\checkmark$ \\
\hline Regulation of cellular metabolic process & $\checkmark$ & . & . & $\checkmark$ & $\checkmark$ & . & $\checkmark$ & & $\checkmark$ & $\checkmark$ \\
\hline Regulation of primary metabolic process & $\checkmark$ & . & . & $\checkmark$ & $\checkmark$ & & $\checkmark$ & & $\checkmark$ & $\checkmark$ \\
\hline Regulation of macromolecule metabolic process & $\checkmark$ & & & $\checkmark$ & $\checkmark$ & . & $\checkmark$ & . & $\checkmark$ & $\checkmark$ \\
\hline Regulation of metabolic process & $\checkmark$ & 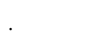 & . & $\checkmark$ & $\checkmark$ & . & $\checkmark$ & & $\checkmark$ & $\checkmark$ \\
\hline Response to chitin & $\checkmark$ & $\checkmark$ & . & $\checkmark$ & . & & $\checkmark$ & & $\checkmark$ & $\checkmark$ \\
\hline Regulation of cellular process & $\checkmark$ & & . & $\checkmark$ & $\checkmark$ & . & $\checkmark$ & & $\checkmark$ & $\checkmark$ \\
\hline Response to osmotic stress & $\checkmark$ & r. & . & $\checkmark$ & $\checkmark$ & . & $\checkmark$ & . & $\checkmark$ & \\
\hline Response to ethylene stimulus & $\checkmark$ & & 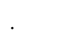 & $\checkmark$ & $\checkmark$ & . & $\checkmark$ & & $\checkmark$ & . \\
\hline Regulation of transcription, DNA-dependent & $\checkmark$ & & & $\checkmark$ & $\checkmark$ & . & $\checkmark$ & . & $\checkmark$ & \\
\hline Regulation of RNA metabolic process & $\checkmark$ & & . & $\checkmark$ & $\checkmark$ & . & $\checkmark$ & & $\checkmark$ & . \\
\hline Chlorophyll biosynthetic process & $\checkmark$ & . & $\checkmark$ & . & . & & $\checkmark$ & $\checkmark$ & $\checkmark$ & . \\
\hline Porphyrin biosynthetic process & $\checkmark$ & & $\checkmark$ & . & . & . & $\checkmark$ & $\checkmark$ & $\checkmark$ & . \\
\hline Tetrapyrrole biosynthetic process & $\checkmark$ & & $\checkmark$ & & & . & $\checkmark$ & $\checkmark$ & $\checkmark$ & \\
\hline Regulation of biological process & $\checkmark$ & & . & $\checkmark$ & . & & $\checkmark$ & & $\checkmark$ & $\checkmark$ \\
\hline
\end{tabular}


Table $3 \mathrm{GO}$ terms attribute matrix from the significantly regulated gene-list for each ecotype, generated using BiNGO software (Continued)

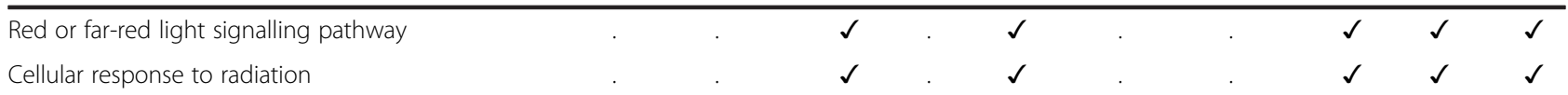

The rows contain different GO terms, and the columns represent 10 ecotypes. A ' $\checkmark$ ' sign represents statistically significant (Hypergeometric test, Benjamini \& Hochberg False Discovery Rate FDR correction, significance level 0.05) overrepresentation of that GO term in corresponding ecotype in that column. Due to space limitations, only some of the interesting GO terms overrepresented in multiple ecotypes were included in this table. The complete result obtained from the BiNGO analysis has been presented in the Additional file 3.

At3g56940 (CRD1), At4g34740 (CIA1), At1g78600 (STH3), At1g71030 (MYBL2), and At5g67030 (ZEP). Havaux et al. [56] reported that $A$. thaliana was able to survive in cold stress through light independent xanthophyll cycle by illustrating protective functions of carotenoid and flavonoid pigments against excess visible radiation at cold temperature [56]. Cold stress also induces synthesis of early light-induced proteins (ELIPs) [57]. Low temperature induces the accumulation of various antioxidants including carotenoids (except $\beta$-carotene), vitamin $\mathrm{E}$ $(\alpha-$ and $\gamma$-tocopherol) and non-photosynthetic pigments (anthocyanins and other flavonoids) [58]. Genes in the overrepresented category pigment biosynthetic process from our analysis support the previous reports.

\section{Cold stress and circadian rhythms}

Circadian rhythm is one of the most prominent overrepresented categories in our dataset. It included many wellknown genes belong to this category like At1g22770 (GI), At1g68050 (FKF1), At1g18330 (RVE7), At5g24470 (PRR5) [59], At5g17300 (RVE1), At2g46790 (TL1), and At2g46830 (CCA1). Previous studies reported a circadian clock regulated induction of $C B F$ genes during low-temperature treatment in $A$. thaliana plants $[60,61]$. The circadian clock gates both gene expression and physiological responses to low R/FR during rapid shade avoidance $[62,63]$. Mikkelsen et al. [64] reported that cold- and clock-regulated gene expression are integrated through regulatory proteins that bind to Evening Element (EE) and Evening Element Like (EEL) elements supported by transcription factors acting at ABA response element (ABREL) sequences [64]. They established a role for circadian evening elements in coldregulated gene expression in $A$. thaliana. Our current results are in good agreement with these previous reports.

\section{Co-regulation of cold and biotic stress responsive genes}

Few categories in our gene set enrichment analysis (GSEA) were related to biotic stress response processes. Some of these categories were as follows -response to other organism, response to fungus, and response to bacterium, multi-organism process etc. Some of the up-regulated genes in these categories include At2g40140 (ZFAR1), At5g25110 (CIPK25), At5g25910 (RLP52), At1g20440 (RD17/COR47), At4g37150 (MES9), At3g50970 (XERO2), At2g42530 (COR15B), At2g44490 (PEN2), At5g64750
(ABR1), At1g51090, At4g12470, At4g36010 (pathogenesisrelated thaumatin family protein), At3g51660 (MIF family protein), At1g20030 (pathogenesis-related thaumatin family protein), At3g50260 (CEJ1), At3g15210 (RAP2.5), At5g58600 (PMR5), At3g52400 (SYP122), At3g06490 (MYB108), At1g19180 (TIFY10A), At4g23810 (WRKY53). Additional file 4 contains all the GO categories from each of ecotypes including the ecotype specific categories. One important observation was that biotic stress response related categories- response to other organism, response to fungus, response to bacterium, response nematode were overrepresented mainly in the southern ecotypes like Cvi, Kas1, Kyo2, Kond. Probable reason may be that plants from southern latitude often face such biotic invaders compare to their northern counterparts, and consequently have coevolved with better and prompt defence response mechanisms against them. Based on genetic resources of $A$. thaliana, coupled with 39 years of field data, it has been reported that natural enemies drive geographic variation in plant defences [65].

Unlike cold tolerance, molecular mechanism of pathogen resistance obtained through cold treatment is not understood well. Plazek et al. reported that cold treatment on spring barley (Hordeum vulgare.), meadow fescue (Festuca pratensis) and oilseed winter rape (Brassica napus var. oleifea) induced resistance to their specific pathogens [66]. Zhu et al. identified a plant temperature sensitive component in disease resistance and provided a potential means to generate plants adapting to a broader temperature range [67]. Besides the available reports about enhanced disease resistance acquired through cold treatment, it is not yet known if these two traits are regulated by the same signal transduction pathway [68]. We have observed overrepresentation of GO categories like steroid hormone mediated signalling pathway, brassinosteroid mediated signalling pathway, jasmonic acid stimulus etc. Phytohormones are involved in induction of disease resistance upon pathogen infection. Plant hormones like salicylic acid (SA) ethylene (ET), jasmonic acid (JA) pathways are known to play important functions in the signal transduction during biotic stresses [69]. The occurrence of simultaneous biotic and abiotic stresses increases the complexity, as the responses to these are largely controlled by different hormone signalling pathways that may interact and inhibit one another 
[70]. Interaction of cold temperature and pathogen attack results potentially negative impact on plants [71]. Plants grow in heavy snowfall areas need to enhance disease resistance to survive from the attack of pathogens like snow molds [72]. Hence, as a nascent observation, the co-evolution of regulatory mechanism for cooccurring stress related genes and processes are highly possible. Further targeted screening of more ecotypes may find interesting results in this direction to explore interaction of biotic and abiotic stress on adaptive evolution of plant defence response.

\section{CBF regulon genes exhibits differential expression pattern in Arabidopsis ecotypes during cold treatment}

The $A$. thaliana CBF cold response pathway has a major role in cold response. $C B F$ genes appear to be present across plant species and are almost always present as a gene family. In $A$. thaliana, there are four characterized $C B F$ genes: $C B F 1,2$ and 3, located on chromosome 4, are cold induced; except CBF4 located on chromosome 5, which is reported to be involved in drought tolerance $[73,74]$. All the $C B F$ genes as well as the selected COR genes were cold regulated in 10 ecotypes. But we observed different levels of expression of $C B F$ and $C O R$ genes in the ten ecotypes. All the $C B F$ genes were induced but $C O R$ genes had preferential expressions in different ecotypes (Figure 2). DREBA1 expression consistently occurred in all the accessions. In a previously published study $C B F 1$ and $C B F 2$ were reported to have quite comparable expression levels in 9 ecotypes except low expression of both in Cvi [17]. Low expression of $C B F 1,2$ in Cvi ecotype is clearly visible in our data (Figure 2). It was reported that expression of the CBF1, 2 and 3 genes was not correlated with cold tolerance level among ecotypes [59].

Variation in gene expression reflects the interplay between 'robustness' and 'evolvability'; which is generally achieved by regulatory divergence. An organism has to preserve a consistent function under different conditions and at the same time, it needs to sustain the ability to evolve in order to adapt to new environments. The plasticity of gene expression may be achieved by selective accumulation of mutations in the promoter. As about 100 downstream genes and processes are regulated by the $\mathrm{CBF}$ and COR proteins, difference could be seen in the expression pattern of down-stream genes which was visible in the heat-map of 1000 genes and other ecotype specific differentially regulated genes (Figure 1). We chose to investigate the polymorphism present in the CBF1, 2 and 3 genes and few $C O R$ genes using recently released data from $A$. thaliana 1001 genome project [75].

\section{Sequence polymorphisms seen in the CBF genes using data from Arabidopsis thaliana 1001 genome project}

Sequence variation of $C B F$ and $C O R$ genes could exert an effect at two different levels: either in the expression of the $C B F$ genes themselves, via polymorphism in the respective promoter and/or in the expression of their downstream genes. It has been reported earlier that all three $C B F$ genes were highly polymorphic, particularly in their promoters, with $C B F 1$ the most and $C B F 2$ the least polymorphic gene $[17,76]$. Hence, we have downloaded the sequence data (including $1 \mathrm{kbp}$ upstream of the coding region) available from the 1001 genome database and calculated Tajima's D statistic to evaluate the allele frequency spectrum and quantify the excess of rare alleles. We observed significant number of non-synonymous amino acid changes in the coding region of the $C B F$ genes (Additional file 5). The three $C B F \mathrm{~s}$ have shown significantly negative Tajima's $\mathrm{D}$ values $(C B F 1=-1.291, C B F 2=-2.223, D R E B A 1=-2.165)$. More negative and significantly lower values of Tajima's D indicate an excess of rare and recent, alleles [45]. But, it is known from earlier studies that the average distribution of

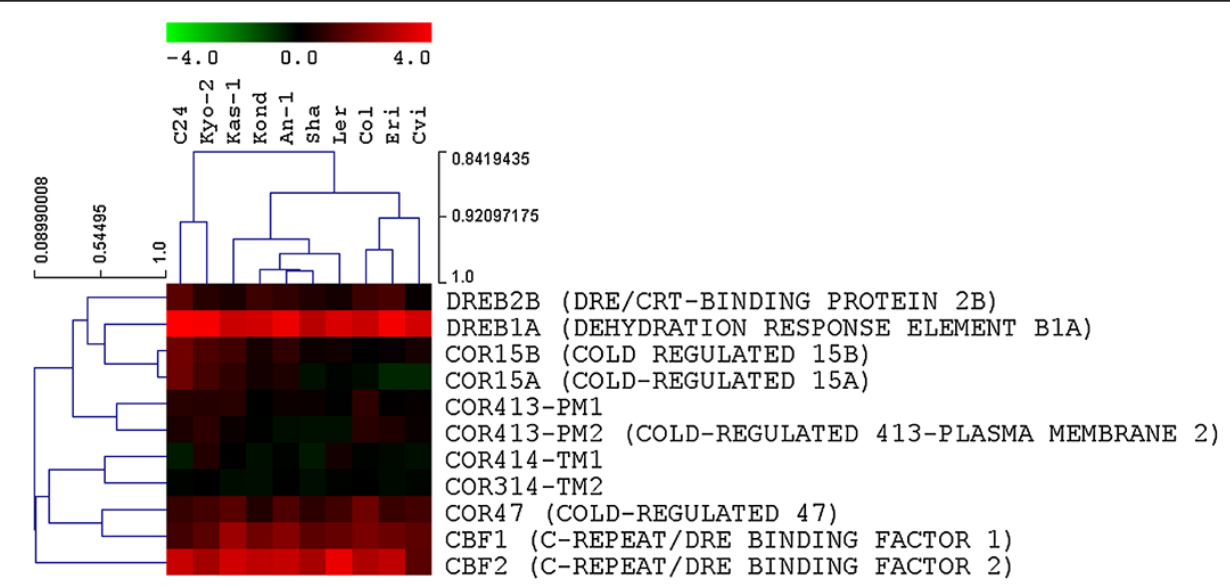

Figure 2 Difference in gene expression among the CBF and COR genes. CBF genes as well as the selected COR genes were cold regulated in all accessions. But there were noticeable differences in the levels of expression among ten $A$. thaliana ecotypes. 
Tajima's $\mathrm{D}$ in the $A$. thaliana genome is known to be biased towards negative values [77-79]. We could not conclude any direct correlation between sequence polymorphism on gene expression pattern of the $C B F$ and $C O R$ genes. The non-existence of a clear correlation between $C B F$ and $C O R$ gene expression with sequence polymorphism in 10 ecotypes might have several reasons. There are other CBF independent pathways and their complex interactions between different components contribute to cold tolerance [12]. So, how these complex interactions of other pathways affect $C B F$ and $C O R$ gene expression would be difficult to predict. Again, COR genes are often up-regulated much later, and this is also true for protein expression. Gene expression without protein synthesis can not generate downstream product. Apart from genotype variation, the length of cold exposure and treatment temperature also affect the gene expression level that leads to freezing tolerance [80]. While studying natural variation of transcriptional auxin response networks in A. thaliana, Delker et al. reported that differentially regulated signalling networks had a greater role to play than sequence polymorphism [81]. Considering such facts, we wanted to explore the pattern of regulatory divergence of cold stress response network among these ten $A$. thaliana ecotypes.

\section{Reverse engineering transcriptional regulatory network during cold stress response in A. thaliana}

Due to the lack of experimentally validated transcriptional regulatory information in A. thaliana, we have decided to reverse engineer an in-silico transcriptional regulatory network model during cellular responses to cold stress in $A$. thaliana using our gene expression data. For this purpose, we have selected top 1,509 differentially cold regulated transcripts from the union of the entire cold regulated transcripts list, considering a criterion that a transcript had to be significantly regulated at least in 2 of the 10 ecotypes. The resulting list contained 178 TFs and 1,331 target genes (TGs). By using NCA method (explained in materials and methods), we have constructed the network at correlation-coefficient threshold $\geq 0.8$. Activation and repression interactions were calculated using the positive and negative correlations. The resulting network contained 1,275 nodes and 7,720 connections; out of them 6,731 connections were activations (positive) and 989 were repressions (negative) (Additional file 6 and Figure 3A). Some of the highly connected positive regulators (TFs) were ATTLP7, POSF21, AS1, RTV1, APRR9, BT1, ANAC102, ANAC035, GLK2, ZFN1, WRKY11, HAC5, MYB73, DA1, LBD41, SR1, WRKY70. Further details of the constructed network including calculated Pearson's correlation coefficient and $p$-values have been given in Additional file 6 . In the network visualization, transcription factors were marked as green triangles and target genes were marked as red circles. General network topology based analysis has revealed that the network exhibited power-law degree distribution [82] (Figure 3B). We have also calculated several other graph-theoretic parameters as described by Barabasi et al. [83]. Some of the parameters were as follows- clustering coefficient $=0.3$, connected components $=3$, network diameter $=11$, characteristic path length $=3.67$, average number of neighbours $=$ 11.385. The constructed network satisfies the existing notion about scale free behaviour of biological networks [84]. Few of the TFs in the network are highly connected (hubs) than others. The generated network (.cys file) has been provided as Additional file 7. Interested reader can locally open the file using Cytoscape software [85] and do more interactive exploration. The presented view of the annotated network in this manuscript has been simplified manually.

Correlation between activity and expression values of TFs Simple correlation between the expression profile of a transcription factor and its targets is not obvious, and simple clustering based methods have not been very successful in deciphering them [86]. The key assumption during prediction of interactions between TFs and their target genes using gene expression data is that high dimensional mRNA expression profiles contain hidden regulatory signals, which can be decomposed to lowdimensional regulatory signals driven through an interacting network [87]. The lower dimensional regulatory signals can be represented as a bipartite networked system of the transcription factors and the target genes, where the gene expression levels are transformed into weighted functions of the intracellular states corresponding to the activity of the transcription factors $[31,33]$. Several such methodologies have been used in plant systems to infer regulatory relationships at various occasions [23,33,88-91].

The NCA algorithm requires two inputs to calculate the hidden regulatory activity profiles: a series of gene expression profiles and a pre-defined regulatory network. The $A$. thaliana transcription factor list were collected from the Database of Arabidopsis Transcription Factors (DATF) [25], The Arabidopsis Gene Regulatory Information Server (AGRIS) [26], and the Plant Transcription Factor Database (PlantTFDB) [92]. List of 59 cold regulated transcription factors were collected from Gene Ontology database under the annotation category 'response to cold' [43]. The constructed network was able to capture $30(\sim 50 \%)$ of these already reported cold regulated TFs.

\section{Transcription factor activity under cold stress in different Arabidopsis ecotypes}

We have compared the predicted activities of the 30 previously reported cold responsive transcription factors 


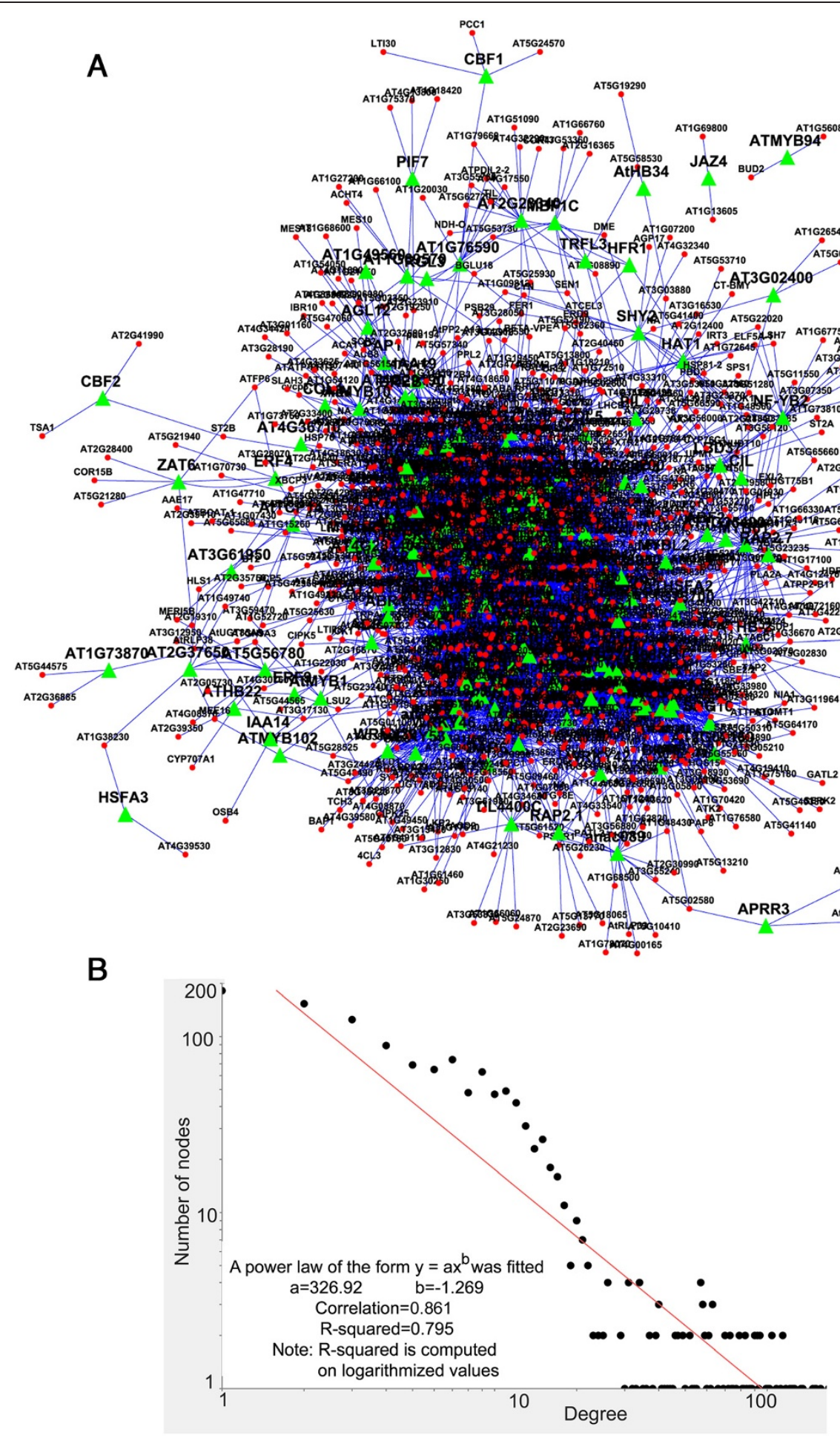

Figure 3 Transcriptional regulatory network constructed using cold stress microarray data from 10 A. thaliana ecotypes. (A) Topological overview of the constructed network. The network contains 1,275 genes (nodes) and 7,720 connections. Transcription factors are marked as green triangles and target genes are marked as red circles. Predicted regulatory interactions are shown as arrow $(\rightarrow)$ for activation $(6,731)$ and down-horizontal bar ( () as repression (989). Network was visualized in Cytoscape software using 'Force-Directed Layout'. (B) Scale-free behaviour of the predicted network. This plot shows the power-law degree distribution of the network $P(k)$ at correlation thresholds $(r \geq 0.8)$. Here $k$ indicates connectivity, and $P(k)$ indicates the connectivity distribution of the genes (nodes) in the network. This satisfies the existing notion about scale free behaviour of biological networks. Few TFs in the network are highly connected (hubs) than others. 
with their corresponding gene expression values in the ten A. thaliana ecotypes. About $57 \%$ of transcription factors showed moderate correlation (Pearson correlation coefficient, $|\mathrm{r}|>0.5)$ between their activities and expressions (Figure 4). Thirteen TFs ((ZFAR1 (At2G40140), At1G 28050, ERF2 (At5G47220), ZAT10 (At1G27730), At5G 46710, CRF4 (At4G27950), At1G78700, At5G17300, At4G28140, At5G48250, At4G29190, RAP2 (At1G46768), and WRKY7 (At4G24240)) exhibited positive correlations ( $r>0.5$ ). For instance CZF-1 (At2G40140) had correlation $r=0.9206$ which indicated that there might be chance of auto regulation. This assumption was supported by literature [93] and information available from the Arabidopsis Gene Regulatory Information Server (AGRIS). Four transcription factors ((WRKY25 (At2G30250), ERF6 (At4G17490), DREB2B (At3G11020) and TIFY10A (At1G 19180)) showed strong negative correlation $(\mathrm{r}<-0.5)$ and the remaining TFs displayed low or no correlation at all $(|\mathrm{r}|<0.5)$. Three of these predictions have been confirmed from AGRIS database.

The predicted activity profiles of thirty cold regulated TFs have clearly shown the ecotype specific variations in the ten $A$. thaliana ecotypes (Figure 4). For example, transcription factor At5G17300 (RVE1) was highly active in Eri, C24 and Col ecotypes compared to the others. Most of the transcription factors were active in more than two ecotypes (Table 4). We have also identified ecotype specific transcription factors (highly active in single ecotype). Transcription factors At1G04240 (SHY2), At2G46830 (CCA1) and At3G11020 (DREB2B) were active in Sha ecotype and transcription factor At4G25490 (DREB1B/CBF1) was more active in Eri ecotype. Spatiotemporal regulatory dynamics of $S H Y 2$ [94] and CCA1 $[95,96]$ have been reported earlier. The transcription factor At5G17490 (RGL3) [97] was active in Col-0 ecotype. We found a group of transcription factors, which were highly active in a particular set of ecotypes. For example, transcription factors At1G27730 (ZAT10), At1G28050, At3G17609 $(H Y H)$, AtAt4G27950 (CRF4), At5G17300 and At5G48250 were active in Eri, C24 and Col-0 ecotypes and transcription factors At1G9180 and At4G25480 were highly responsive in Eri and Col-0 ecotypes during cold treatment. All the A. thaliana ecotypes had at least 7 (out of 30 core cold responsive TFs) active TFs, except the Kond ecotype. This ecotype had only two significantly active TFs (At1G76590 and At4G04450).

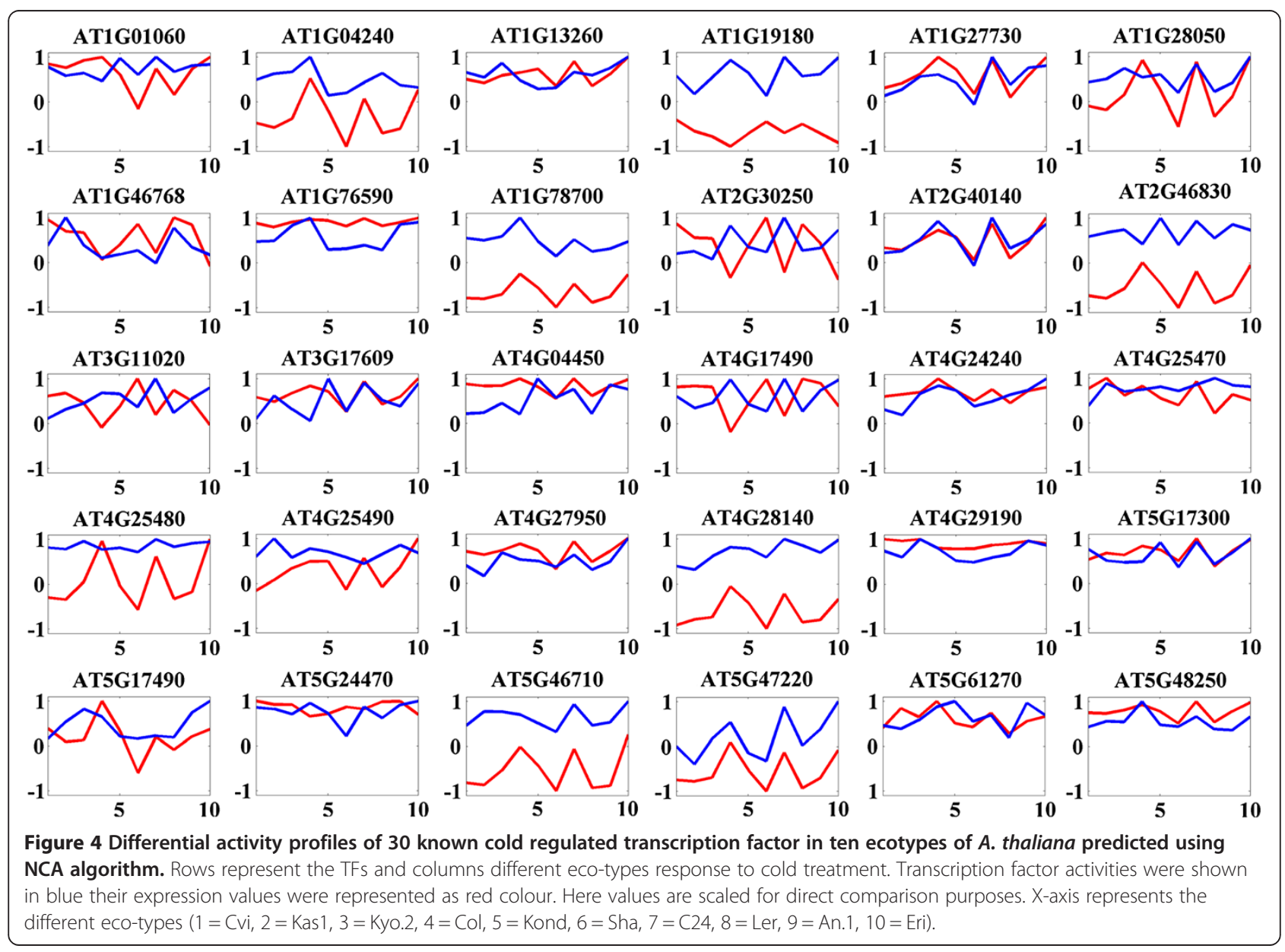


Table 4 Ecotype specific transcriptional activity profiles of the 30 cold responsive TFs

\begin{tabular}{|c|c|c|}
\hline TF ID & Alias & Ecotypes \\
\hline At1G01060 & LHY1 & Eri, Col-0, Cvi, Kyo-2 \\
\hline At1G04240 & $\mathrm{SHY} 2$ & Sha \\
\hline At1G13260 & RAV1 & Eri, C24 \\
\hline At1G19180 & TIFY10A & Eri, Col-0 \\
\hline At1G27730 & ZAT10 & Eri, C24, Col-0 \\
\hline At1G28050 & At1G28050 & Eri, C24, Col-0 \\
\hline At1G46768 & RAP2.1 & An-1, Sha, Ler, Cvi \\
\hline At1G76590 & At1G76590 & Eri, Kond, C24, An-1, Col-0, Sha, Ler, Cvi, Kyo-2, Kas-1 \\
\hline At1G78700 & At1G78700 & Sha, Ler, Cvi, Kas-1 \\
\hline At2G30250 & WRKY25 & Sha, Ler, Cvi \\
\hline At2G40140 & ZFAR1/CZF1 & Eri, C24 \\
\hline At2G46830 & CCA1 & Sha \\
\hline At3G11020 & DREB2B & Sha \\
\hline At3G17609 & $\mathrm{HYH}$ & Eri, C24, Col-0 \\
\hline At4G04450 & WRKY42 & Eri, Kond, C24, An-1, Col-0, Cvi, Kyo-2, Kas-1 \\
\hline At4G17490 & ERF-6-6 & An-1, Sha, Ler, Cvi, Kyo-2, Kas-1 \\
\hline At4G24240 & WRKY7 & Eri, Col-0 \\
\hline At4G25470 & CBF2 & C24, Col-o, Kas-1 \\
\hline At4G25480 & DREB1A & Eri, Col-0 \\
\hline At4G25490 & DREB1B/CBF1 & Eri \\
\hline At4G27950 & CRF4 & Eri, C24, Col-0 \\
\hline At4G28140 & At4G28140 & An-1, Sha, Ler, Cvi, Kas-1 \\
\hline At4G29190 & At4G29190 & Eri, An-1, Cvi, Kyo-, Kas-1 \\
\hline At5G17300 & At5G17300 & Eri, C24, Col-0 \\
\hline At5G17490 & RGL3 & Col-0 \\
\hline At5G24470 & PRR5 & An-1, Ler, Cvi, Kyo-2, Kas-1 \\
\hline At5G46710 & At5G46710 & An-1, Sha, Ler, Cvi, Kas-1 \\
\hline At5G47220 & ERF2 & Sha, Ler, Kas-1 \\
\hline At5G48250 & At5G48250 & Eri, C24, Col-0 \\
\hline At5G61270 & PIF7 & Col-0, Kas-1 \\
\hline
\end{tabular}

This table presents, which of the 30 previously reported cold responsive TFs are active among ten ecotypes during our experiments based on their predicted activity profiles using NCA algorithm.

\section{Conclusions}

Here we undertook an experiment to analyze the natural variation in genome-scale cold stress response regulatory networks in ten $A$. thaliana ecotypes at a single time point ( 3 hours) gene expression measurement. The analysis indicated that the $10 \mathrm{~A}$. thaliana ecotypes had different transcriptome level signatures in response to nonfreezing cold stress. Col-0 being known as cold tolerant ecotype had significantly less number of differentially regulated transcripts while Cvi as the most southern most ecotype had the highest number of differentially regulated transcripts. Among the differentially cold regulated transcripts, $75 \%$ showed ecotype specific expression pattern. There were 315 transposable elements
(TEs) in the ecotype specific differentially regulated gene list. These TEs may play an important role in plant genome evolution while adapting to local climatic temperatures. $C B F$ genes were cold induced in all the ecotypes, irrespective of their geographic origin. But their levels of expressions varied among different ecotypes. Expression pattern of the COR genes were not consistent in all ecotypes. Sequence data available from the 1001-genome project indicated that the mutations in their sequences might contribute to the dramatic difference in the expression pattern. Significant numbers of non-synonymous amino acid changes were observed in the coding region of all the $C B F$ genes. All of the $C B F \mathrm{~s}$ had shown significantly negative Tajima's $\mathrm{D}$ values, indicating an excess of rare and 
recent, alleles. Gene ontology analysis had shown that apart from common cold stress regulated processes; several other biological processes were differentially regulated in the 10 ecotypes. Some of the important GO categories were - pigment biosynthesis, circadian rhythm, response to light, response to water deprivation, response to ABA. By looking at the differentially regulated genes related to pathogen responses induced by cold stress, a primary assumption was made that the co-evolution of severely affecting co-occurring stress related genes and processes was highly possible. We have constructed an in silico transcriptional regulatory network model during cellular responses to non-freezing cold stress in A. thaliana, using gene expression data from 10 ecotypes. The network contained 1,275 nodes and 7,720 connections, which included 178 TFs and 1,331 target genes. Apart from retaining several previously known interactions (cross validated using AGRIS), many novel regulatory interactions during the cold stress response in A. thaliana were suggested. Differential regulatory activities were observed among the cold regulated TFs, which might contribute towards cold adaptation of the ecotypes. In addition, since the approach is general, it could in principle be used to study networks regulating biological process in any biological systems. As far as cold stress is concerned, it could be implemented for identification of useful molecular markers or relevant forward genetics experiments to develop cold tolerant crop varieties.

\section{Additional files}

Additional file 1: List of all transcripts from 10 ecotypes with annotations, $p$-values and fold change values during cold treatment. United list of 6061 differentially cold regulated transcripts and 498 TFs are in separate sheets of the same excel file.

Additional file 2: List of statistically significant ecotype specific gene expressions during cold treatment from 10 ecotypes are presented in 10 different sheets.

Additional file 3: Results of Gene Set Enrichment Analysis using BinGO software. The individual results for each of the 10 ecotypes have been put in a single file. Each analysis contains the detailed statistical test, significance score for each GO-term and corresponding gene IDs in that category.

Additional file 4: $\mathrm{GO}$ category contingency table from the significantly regulated gene-list, generated using BiNGO software. The rows contain different $\mathrm{GO}$ terms, and the columns represent 10 ecotypes. A ' $\checkmark$ ' sign represents statistically significant (Hypergeometric test, Benjamini \& Hochberg False Discovery Rate FDR correction, significance level 0.05 ) overrepresentation of that $\mathrm{GO}$ term in corresponding ecotype in that column. The column with the header 'occurrence count' represents in how many ecotypes the respective term is significantly over represented.

Additional file 5: Analysis of sequence polymorphism in CBF and COR genes.

Additional file 6: TF-TG regulatory bipartite connections predicted using NCA algorithm based on their activity profiles, using Pearson correlation coefficient threshold $(P C C) \geq 0.80$. The second sheet (TF_ACT_REP) includes predicted pattern of regulation (activation or repression) in the network.
Additional file 7: Predicted TF-TG regulatory networks as a .cys file, which can be open locally by a reader for interactive exploration. For visualizing the network locally, download cytoscape software from http://www.cytoscape.org/ and load the .cys files on the software. The presented view of the annotated network in this manuscript has been simplified manually for representation purpose.

Competing interests

The authors declare that they have no competing interests.

\section{Authors' contributions}

$J \mathrm{M}, \mathrm{AMB}$ and $\mathrm{HBN}$ conceived the multistress project. PB developed the concept of the study, performed bioinformatics analyses and drafted the manuscript. NDJ performed the NCA analysis. SR and HBN were responsible for the microarray experiments and data pre-processing. JM was leading the ERA-NET PG Multi-Stress project and contributed towards improvement of the manuscript. AMB coordinated the overall development of the manuscript. All authors have read and approved the manuscript.

\section{Acknowledgments}

We sincerely acknowledge the funding from ERA-NET Plant Genomics (http://www.erapg.org/) for Multi-Stress project. The arrays experiments were run at the DTU Multi-Assay Core (DMAC) thanks to an infrastructure grant from the Technical University of Denmark. PB was supported by the Biotechnology and Functional Genomics (FUGE) program of the Norwegian Research Council through grants NFR 184147/S10, 185173/N40, 184146/S10 and NDJ was supported by the Norwegian Research Council, through grant number 7017430. We thank Drs. Jens Rohloff, Richard Strimbeck, Christophe Pelabon and Nadav Skjøndal-Bar for critical reading of the manuscript.

\section{Author details}

'Department of Biology, Norwegian University of Science and Technology, Trondheim N-7491, Norway. ${ }^{2}$ Department of Chemical Engineering, Norwegian University of Science and Technology, Trondheim N-7491, Norway. ${ }^{3}$ Center for Biological Sequence Analysis, Department of Systems Biology, Technical University of Denmark, Lyngby DK 2800, Denmark. ${ }^{4}$ Department of Biology, University of Copenhagen, Copenhagen DK-2200, Denmark.

Received: 4 June 2013 Accepted: 11 October 2013

Published: 22 October 2013

\section{References}

1. Hall BK, Hallgrímsson B, Strickberger MW: Strickberger's evolution: the integration of genes, organisms and populations. 4th edition. Sudbury, Mass: Jones and Bartlett; 2008.

2. Alonso-Blanco C, Aarts MGM, Bentsink L, Keurentjes JJB, Reymond M, Vreugdenhil $\mathrm{D}$, Koornneef $\mathrm{M}$ : What has natural variation taught us about plant development, physiology, and adaptation? Plant Cell 2009, 21:1877-1896.

3. Mitchell-Olds T, Schmitt J: Genetic mechanisms and evolutionary significance of natural variation in Arabidopsis. Nature 2006, 441:947-952.

4. Horton MW, Hancock AM, Huang YS, Toomajian C, Atwell S, Auton A, Muliyati NW, Platt A, Sperone FG, Vilhjalmsson BJ, et al: Genome-wide patterns of genetic variation in worldwide Arabidopsis thaliana accessions from the RegMap panel. Nat Genet 2012, 44:212-216.

5. Alonso-Blanco C, Koornneef M: Naturally occurring variation in Arabidopsis: an underexploited resource for plant genetics. Trends Plant Sci 2000, 5:22-29.

6. Sanghera GS, Wani SH, Hussain W, Singh NB: Engineering cold stress tolerance in crop plants. Curr Genomics 2011, 12:30-43.

7. Thomashow MF: Plant cold acclimation: freezing tolerance genes and regulatory mechanisms. Annu Rev Plant Physiol Plant Mol Biol 1999, 50:571-599.

8. Penfield S: Temperature perception and signal transduction in plants. The New phytologist 2008, 179:615-628.

9. Carvallo MA, Pino MT, Jeknic Z, Zou C, Doherty CJ, Shiu SH, Chen TH, Thomashow MF: A comparison of the low temperature transcriptomes and $\mathrm{CBF}$ regulons of three plant species that differ in freezing tolerance: Solanum commersonii, Solanum tuberosum, and Arabidopsis thaliana. J Exp Bot 2011, 62:3807-3819. 
10. Yamaguchishinozaki $K$, Shinozaki $K$ : A novel Cis-acting element in an Arabidopsis gene is involved in responsiveness to drought, low-temperature, or high-salt stress. Plant Cell 1994, 6:251-264.

11. Jaglo-Ottosen KR, Gilmour SJ, Zarka DG, Schabenberger O, Thomashow MF: Arabidopsis CBF1 overexpression induces COR genes and enhances freezing tolerance. Science 1998, 280:104-106.

12. Fowler $\mathrm{S}$, Thomashow MF: Arabidopsis transcriptome profiling indicates that multiple regulatory pathways are activated during cold acclimation in addition to the CBF cold response pathway. Plant Cell 2002, 14:1675-1690.

13. Swindell WR, Huebner M, Weber AP: Plastic and adaptive gene expression patterns associated with temperature stress in Arabidopsis thaliana. Heredity 2007, 99:143-150.

14. Rohde P, Hincha DK, Heyer AG: Heterosis in the freezing tolerance of crosses between two Arabidopsis thaliana accessions (Columbia- 0 and C24) that show differences in non-acclimated and acclimated freezing tolerance. Plant Journal 2004, 38:790-799.

15. Alonso-Blanco C, Gomez-Mena C, Llorente F, Koornneef M, Salinas J, Martinez-Zapater JM: Genetic and molecular analyses of natural variation indicate CBF2 as a candidate gene for underlying a freezing tolerance quantitative trait locus in Arabidopsis. Plant Physiol 2005, 139:1304-1312.

16. Zhen $Y$, Ungerer MC: Clinal variation in freezing tolerance among natural accessions of Arabidopsis thaliana. New Phytologist 2008, 177:419-427.

17. McKhann HI, Gery C, Berard A, Leveque S, Zuther E, Hincha DK, De Mita S, Brunel D, Teoule E: Natural variation in CBF gene sequence, gene expression and freezing tolerance in the Versailles core collection of Arabidopsis thaliana. BMC Plant Biol 2008, 8:105.

18. Somerville $C$, Koornneef $M$ : A fortunate choice: the history of Arabidopsis as a model plant. Nat Rev Genet 2002, 3:883-889.

19. Moreau Y, Aerts S, De Moor B, De Strooper B, Dabrowski M: Comparison and meta-analysis of microarray data: from the bench to the computer desk. Trends Genet 2003, 19:570-577.

20. Rasmussen $S$, Barah $P$, Suarez-Rodriguez MC, Bressendorff S, Friis $P$ Costantino P, Bones AM, Nielsen HB, Mundy J: Transcriptome responses to combinations of stresses in Arabidopsis. Plant Physiol 2013, 161:1783-1794.

21. Austin RS, Vidaurre D, Stamatiou G, Breit R, Provart NJ, Bonetta D, Zhang J, Fung P, Gong Y, Wang PW, et al: Next-generation mapping of Arabidopsis genes. Plant J 2011, 67:715-725.

22. Shimizu KK: Ecology meets molecular genetics in Arabidopsis. Popul Ecol 2002, 44:221-233.

23. Keurentjes JJB, Fu JY, Terpstra IR, Garcia JM, van den Ackerveken G, Snoek LB, Peeters AJM, Vreugdenhil D, Koornneef M, Jansen RC: Regulatory network construction in Arabidopsis by using genome-wide gene expression quantitative trait loci. Proc Natl Acad Sci U S A 2007, 104:1708-1713.

24. Ideker T, Krogan NJ: Differential network biology. Mol Syst Biol 2012, 8:565

25. Guo A, He K, Liu D, Bai S, Gu X, Wei L, Luo J: DATF: a database of Arabidopsis transcription factors. Bioinformatics 2005, 21:2568-2569.

26. Yilmaz A, Mejia-Guerra MK, Kurz K, Liang X, Welch L, Grotewold E: AGRIS: the Arabidopsis Gene Regulatory Information Server, an update. Nucleic acids research 2011, 39:D1118-1122.

27. Tirosh I, Barkai N: Inferring regulatory mechanisms from patterns of evolutionary divergence. Mol Syst Biol 2011, 7:530

28. Segal E, Shapira M, Regev A, Pe'er D, Botstein D, Koller D, Friedman N: Module networks: identifying regulatory modules and their condition-specific regulators from gene expression data. Nat Genet 2003, 34:166-176.

29. Alter O, Brown PO, Botstein D: Singular value decomposition for genome-wide expression data processing and modeling. Proc Natl Acad Sci USA 2000, 97:10101-10106.

30. Herrgard MJ, Covert MW, Palsson BO: Reconstruction of microbial transcriptional regulatory networks. Curr Opin Biotechnol 2004, 15:70-77.

31. Liao JC, Boscolo R, Yang YL, Tran LM, Sabatti C, Roychowdhury VP: Network component analysis: reconstruction of regulatory signals in biological systems. Proc Natl Acad Sci USA 2003, 100:15522-15527.

32. Kao KC, Yang YL, Boscolo R, Sabatti C, Roychowdhury V, Liao JC: Transcriptome-based determination of multiple transcription regulator activities in Escherichia coli by using network component analysis. Proc Natl Acad Sci USA 2004, 101:641-646.

33. Wang J, Qiu X, Li Y, Deng Y, Shi T: A transcriptional dynamic network during Arabidopsis thaliana pollen development. BmC Syst Biol 2011 5(Suppl 3):S8.

34. Irizarry RA, Bolstad BM, Collin F, Cope LM, Hobbs B, Speed TP: Summaries of Affymetrix GeneChip probe level data. Nucleic Acids Res 2003, 31:e15.
35. Carvalho BS, Irizarry RA: A framework for oligonucleotide microarray preprocessing. Bioinformatics 2010, 26:2363-2367.

36. R Core Team: R: A language and environment for statistical computing. In Book R: A language and environment for statistical computing. Edited by City. Vienna, Austria: R Foundation for Statistical Computing; 2012. http:// www.R-project.org/. ISBN 3-900051-07-0.

37. Lamesch P, Berardini TZ, Li D, Swarbreck D, Wilks C, Sasidharan R, Muller R, Dreher K, Alexander DL, Garcia-Hernandez M, et al: The Arabidopsis information resource (TAIR): improved gene annotation and new tools. Nucleic Acids Res 2012, 40:D1202-1210.

38. Guberman JM, Ai J, Arnaiz O, Baran J, Blake A, Baldock R, Chelala C, Croft D, Cros A, Cutts RJ: BioMart central portal: an open database network for the biological community. Database (Oxford) 2011, 2011:bar041.

39. Cui $X$, Churchill GA: Statistical tests for differential expression in CDNA microarray experiments. Genome Biol 2003, 4:210.

40. Kerr MK, Martin M, Churchill GA: Analysis of variance for gene expression microarray data. J Comput Biol 2000, 7:819-837.

41. Saeed Al, Hagabati NK, Braisted JC, Liang W, Sharov V, Howe EA, Li JW, Thiagarajan M, White JA, Quackenbush J: TM4 microarray software suite. Method Enzymol 2006, 411:134-193.

42. Maere S, Heymans K, Kuiper M: BiNGO: a Cytoscape plugin to assess overrepresentation of gene ontology categories in biological networks. Bioinformatics 2005, 21:3448-3449.

43. Ashburner M, Ball CA, Blake JA, Botstein D, Butler H, Cherry JM, Davis AP, Dolinski K, Dwight SS, Eppig JT, et al: Gene ontology: tool for the unification of biology. The Gene Ontology Consortium. Nat Genet 2000, 25:25-29.

44. Chenna R, Sugawara H, Koike T, Lopez R, Gibson TJ, Higgins DG, Thompson JD: Multiple sequence alignment with the Clustal series of programs. Nucleic Acids Res 2003, 31:3497-3500.

45. Tajima F: Statistical-method for testing the neutral mutation hypothesis by DNA polymorphism. Genetics 1989, 123:585-595.

46. Tamura K, Peterson D, Peterson N, Stecher G, Nei M, Kumar S: MEGA5: molecular evolutionary genetics analysis using maximum likelihood, evolutionary distance, and maximum parsimony methods. Mol Biol Evol 2011, 28:2731-2739.

47. Galbraith SJ, Tran LM, Liao JC: Transcriptome network component analysis with limited microarray data. Bioinformatics 2006, 22:1886-1894.

48. Hannah MA, Wiese D, Freund S, Fiehn O, Heyer AG, Hincha DK: Natural genetic variation of freezing tolerance in Arabidopsis. Plant Physiol 2006, 142:98-112.

49. Ziolkowski PA, Koczyk G, Galganski L, Sadowski J: Genome sequence comparison of $\mathrm{Col}$ and Ler lines reveals the dynamic nature of Arabidopsis chromosomes. Nucleic Acids Res 2009, 37:3189-3201.

50. Lee $\mathrm{BH}$, Henderson DA, Zhu JK: The Arabidopsis cold-responsive transcriptome and its regulation by ICE1. Plant Cell 2005, 17:3155-3175.

51. Kashkush K, Feldman M, Levy AA: Gene loss, silencing and activation in a newly synthesized wheat allotetraploid. Genetics 2002, 160:1651-1659.

52. Wang $X$, Weigel $D$, Smith LM: Transposon variants and their effects on gene expression in Arabidopsis. Plos Genetics 2013, 9(2):1-13.

53. Chen WJ, Chang SH, Hudson ME, Kwan WK, Li J, Estes B, Knoll D, Shi L, Zhu T: Contribution of transcriptional regulation to natural variations in Arabidopsis. Genome Biol 2005, 6:R32.

54. Catala R, Medina J, Salinas J: Integration of low temperature and light signaling during cold acclimation response in Arabidopsis. Proc Natl Acad Sci USA 2011, 108:16475-16480.

55. Franklin KA, Whitelam GC: Light-quality regulation of freezing tolerance in Arabidopsis thaliana. Nat Genet 2007, 39:1410-1413.

56. Havaux M, Kloppstech K: The protective functions of carotenoid and flavonoid pigments against excess visible radiation at chilling temperature investigated in Arabidopsis npq and tt mutants. Planta 2001, 213:953-966.

57. Heddad M, Noren H, Reiser V, Dunaeva M, Andersson B, Adamska I: Differential expression and localization of early light-induced proteins in Arabidopsis. Plant Physiol 2006, 142:75-87.

58. Rapacz M, Wolanin B, Hura K, Tyrka M: The effects of cold acclimation on photosynthetic apparatus and the expression of COR14b in four genotypes of barley (Hordeum vulgare) contrasting in their tolerance to freezing and high-light treatment in cold conditions. Ann Bot-London 2008, 101:689-699.

59. Zuther $\mathrm{E}$, Schulz E, Childs LH, Hincha DK: Clinal variation in the non-acclimated and cold-acclimated freezing tolerance of Arabidopsis thaliana accessions. Plant Cell Environ 2012, 35(10):1860-78. 
60. Edwards KD, Anderson PE, Hall A, Salathia NS, Locke JCW, Lynn JR, Straume M, Smith JQ, Millar AJ: Flowering Locus C mediates natural variation in the high-temperature response of the Arabidopsis circadian clock. Plant Cell 2006, 18:639-650.

61. Harmer SL, Hogenesch LB, Straume M, Chang HS, Han B, Zhu T, Wang X, Kreps JA, Kay SA: Orchestrated transcription of key pathways in Arabidopsis by the circadian clock. Science 2000, 290:2110-2113.

62. Salter MG, Franklin KA, Whitelam GC: Gating of the rapid shade-avoidance response by the circadian clock in plants. Nature 2003, 426:680-683.

63. Fowler SG, Cook D, Thomashow ME: Low temperature induction of Arabidopsis CBF1, 2, and 3 is gated by the circadian clock. Plant Physiol 2005, 137:961-968.

64. Mikkelsen MD, Thomashow MF: A role for circadian evening elements in cold-regulated gene expression in Arabidopsis. Plant Journal 2009, 60:328-339.

65. Zust T, Heichinger C, Grossniklaus U, Harrington R, Kliebenstein DJ, Turnbull LA: Natural enemies drive geographic variation in plant defenses. Science 2012, 338:116-119.

66. Plazek A, Zur I: Cold-induced plant resistance to necrotrophic pathogens and antioxidant enzyme activities and cell membrane permeability. Plant Sci 2003, 164:1019-1028.

67. Zhu Y, Qian WQ, Hua J: Temperature modulates plant defense responses through NB-LRR proteins. Plos Pathog 2010, 6(4):1-13.

68. Kuwabara C, Imai R: Molecular basis of disease resistance acquired through cold acclimation in overwintering plants. J Plant Biol 2009, 52:19-26.

69. Fujita M, Fujita Y, Noutoshi Y, Takahashi F, Narusaka Y, Yamaguchi-Shinozaki K, Shinozaki K: Crosstalk between abiotic and biotic stress responses: a current view from the points of convergence in the stress signaling networks. Curr Opin Plant Biol 2006, 9:436-442.

70. Atkinson NJ, Urwin PE: The interaction of plant biotic and abiotic stresses: from genes to the field. J Exp Bot 2012, 63:3523-3543.

71. Mittler R: Abiotic stress, the field environment and stress combination Trends Plant Sci 2006, 11:15-19.

72. Hoshino T, Xiao N, Tkachenko OB: Cold adaptation in the phytopathogenic fungi causing snow molds. Mycoscience 2009, 50:26-38

73. Medina J, Catala R, Salinas J: The CBFs: Three arabidopsis transcription factors to cold acclimate. Plant Sci 2011, 180:3-11.

74. Haake V, Cook D, Riechmann JL, Pineda O, Thomashow MF, Zhang JZ: Transcription factor CBF4 is a regulator of drought adaptation in Arabidopsis. Plant Physiol 2002, 130:639-648.

75. Cao J, Schneeberger K, Ossowski S, Gunther T, Bender S, Fitz J, Koenig D, Lanz C, Stegle O, Lippert C, et al: Whole-genome sequencing of multiple Arabidopsis thaliana populations. Nat Genet 2011, 43:956-963.

76. Zhen $Y$, Ungerer MC: Relaxed selection on the CBF/DREB1 regulatory genes and reduced freezing tolerance in the southern range of Arabidopsis thaliana. Mol Biol Evol 2008, 25:2547-2555.

77. Nordborg M, Hu TT, Ishino Y, Jhaveri J, Toomajian C, Zheng H, Bakker E, Calabrese P, Gladstone J, Goyal R, et al: The pattern of polymorphism in Arabidopsis thaliana. PLOS Biol 2005, 3:e196.

78. Sterken R, Kiekens R, Coppens E, Vercauteren I, Zabeau M, Inze D, Flowers J, Vuylsteke M: A population genomics study of the Arabidopsis core cell cycle genes shows the signature of natural selection. Plant Cell 2009, 21:2987-2998

79. Bakker EG, Toomajian C, Kreitman M, Bergelson J: A genome-wide survey of R gene polymorphisms in Arabidopsis. Plant Cell 2006, 18:1803-1818.

80. Fowler DB, Limin AE: Interactions among factors regulating phenological development and acclimation rate determine low-temperature tolerance in wheat. Ann Bot-London 2004, 94:717-724.

81. Delker C, Poschl Y, Raschke A, Ullrich K, Ettingshausen S, Hauptmann V, Grosse I, Quint M: Natural variation of transcriptional auxin response networks in Arabidopsis thaliana. Plant Cell 2010, 22:2184-2200.

82. Clauset A, Shalizi CR, Newman MEJ: Power-Law distributions in empirical data. Siam Rev 2009, 51:661-703.

83. Barabasi AL, Oltvai ZN: Network biology: understanding the cell's functional organization. Nat Rev Genet 2004, 5:101-113.

84. Barabasi AL: Scale-free networks: a decade and beyond. Science 2009 325:412-413.

85. Shannon P, Markiel A, Ozier O, Baliga NS, Wang JT, Ramage D, Amin N, Schwikowski B, Ideker T: Cytoscape: a software environment for integrated models of biomolecular interaction networks. Genome research 2003, 13:2498-2504.
86. Qian J, Lin J, Luscombe NM, Yu H, Gerstein M: Prediction of regulatory networks: genome-wide identification of transcription factor targets from gene expression data. Bioinformatics 2003, 19:1917-1926.

87. Holter NS, Mitra M, Maritan A, Cieplak M, Banavar JR, Fedoroff NV: Fundamental patterns underlying gene expression profiles: simplicity from complexity. Proc Natl Acad Sci USA 2000, 97:8409-8414.

88. Carrera J, Rodrigo G, Jaramillo A, Elena SF: Reverse-engineering the Arabidopsis thaliana transcriptional network under changing environmental conditions. Genome biology 2009, 10:R96.

89. Espinosa-Soto C, Padilla-Longoria P, Alvarez-Buylla ER: A gene regulatory network model for cell-fate determination during Arabidopsis thaliana flower development that is robust and recovers experimental gene expression profiles. The Plant cell 2004, 16:2923-2939.

90. Middleton AM, Farcot E, Owen MR, Vernoux T: Modeling regulatory networks to understand plant development: small is beautiful. The Plant cell 2012, 24:3876-3891.

91. Brady SM, Zhang L, Megraw M, Martinez NJ, Jiang E, Yi CS, Liu W, Zeng A, Taylor-Teeples M, Kim D, et al: A stele-enriched gene regulatory network in the Arabidopsis root. Mol Syst Biol 2011, 7:459.

92. Riano-Pachon DM, Ruzicic S, Dreyer I, Mueller-Roeber B: PInTFDB: an integrative plant transcription factor database. BMC bioinformatics 2007, 8:42.

93. AbuQamar S, Chen X, Dhawan R, Bluhm B, Salmeron J, Lam S, Dietrich RA, Mengiste T: Expression profiling and mutant analysis reveals complex regulatory networks involved in Arabidopsis response to Botrytis infection. Plant J 2006, 48:28-44.

94. Scacchi E, Salinas P, Gujas B, Santuari L, Krogan N, Ragni L, Berleth T, Hardtke CS: Spatio-temporal sequence of cross-regulatory events in root meristem growth. Proc Natl Acad Sci USA 2010, 107:22734-22739.

95. Li G, Siddiqui H, Teng Y, Lin R, Wan XY, Li J, Lau OS, Ouyang X, Dai M, Wan J, et al: Coordinated transcriptional regulation underlying the circadian clock in Arabidopsis. Nat Cell Biol 2011, 13:616-622

96. Wang W, Barnaby JY, Tada Y, Li H, Tor M, Caldelari D, Lee DU, Fu XD, Dong X: Timing of plant immune responses by a central circadian regulator. Nature 2011, 470:110-114.

97. Feng S, Martinez C, Gusmaroli G, Wang Y, Zhou J, Wang F, Chen L, Yu L, Iglesias-Pedraz JM, Kircher S, et al: Coordinated regulation of Arabidopsis thaliana development by light and gibberellins. Nature 2008, 451:475-479.

doi:10.1186/1471-2164-14-722

Cite this article as: Barah et al.: Genome-scale cold stress response regulatory networks in ten Arabidopsis thaliana ecotypes. BMC Genomics $201314: 722$

\section{Submit your next manuscript to BioMed Central and take full advantage of:}

- Convenient online submission

- Thorough peer review

- No space constraints or color figure charges

- Immediate publication on acceptance

- Inclusion in PubMed, CAS, Scopus and Google Scholar

- Research which is freely available for redistribution 